PACS: 42.79.Pw, 68.55 Ac

\title{
Features of growing epitaxial layers from solid solutions based on indium and aluminium arsenides
}

\author{
D.M. Yodgorova, A.V. Karimov \\ Physical-Technical Institute of the Scientific Association "Physics-Sun" of the Academy of Sciences of the Republic \\ of Uzbekistan, Tashkent, Mavlanova 2b, Tashkent 700084. (fax 998+71+1354291). \\ E-mail:karimov@physic.uzsci.net
}

\begin{abstract}
Growing the epitaxial layers that contain the aluminium or indium has its specific features. So, the epitaxial layers prepared using the method of liquid-phase epitaxy at high ( 0.1 to 0.2 at. \%) contents of a replacing element can possess a phase non-uniform boundary interface or goffered surface. Height of peaks can run from 2 up to $15 \mu \mathrm{m}$ in them. The results of researches of diode structures based on epitaxial layers obtained by a combined method are represented in this work. The difference of the used method is that in the beginning growth of a layer goes as that under compulsory cooling, and further as the combined one. So, the melt will be combined due to the solid phase from the epitaxial layer, after admission of the first portion of a solution-melt onto the substrate when cooling the system and another part of the solution-melt will become saturated. In the beginning, the layer grows due to isothermal epitaxy caused by mixing the first and second portions of melts when feeding the next discrete portion of the solution-melt, onto the previous one. After their composition equalization, there takes place a growth caused by compulsory cooling. This way to grow the epitaxial layers can be considered as delta-thermal liquid-phase epitaxy in the multistage process. $\Delta T=3 \ldots 10^{\circ} \mathrm{C}$ is optimum for the initial crystallization temperature $T_{\mathrm{cr}}=750 \ldots 830{ }^{\circ} \mathrm{C}$.
\end{abstract}

Keywords: epitaxial layers, liquid-phase epitaxy, delta-thermal liquid epitaxy.

Manuscript received 28.07.04; accepted for publication 16.12.04.

\section{Introduction}

Semiconductor $p$ - $n$ and metal-semiconductor transitions are a basic element of various semiconductor structures. There the physical processes originate with thin (less than one micrometer) transition and go along a direction of high field area movement. As to properties and characteristics of specific structures, they in many respects depend on parameters of initial materials, technology of preparation and principles of their creation.

One can distinguish the sharp layer structure and that with a dim-diffusion boundaries. At a deviation of connected structure of surfaces structure to some nuclear layers from the bulk structure of contacting materials the sharp borders turn out. Diffusion boundaries comprise several tens of nuclear layers, the structure of which differ from that of the bulk in contacting materials.

At the epitaxial growth due to isothermal epitaxy or compulsory cooling, the epitaxial layers containing aluminium or indium with the content of the replacing element about 0.1 to 0.2 at. \% can posssess a phase nonuniform boundary [1] or goffered surface [2]. The height of peaks in them can reach from 2 up to $15 \mu \mathrm{m}$.
To increase the structural perfection of epitaxial layers (AlGaAs, GaAs, InGaAs), we used the alternate contact of a solution-melt with the grown layer as the multistage process of growth gives the effective results. Thus, the formation of new boundary surfaces preserve dislocations to disseminate from one layer into another. In this aspect, the usage of this way, namely, deltathermal liquid epitaxy [3] in the new updated form is of interest to obtain micro-layer epitaxial films.

The difference of the modified way is that initially the growth of a layer goes similar to that observed at compulsory cooling, but further it is closer to combined process. So, after admission of the first portion $P_{1}$ of the solution-melt onto the substrate, in the course of cooling the system to the chosen temperature $T_{1}=T_{0}-T_{1}$, the melt will be exhausted for the sake of coming down of the epitaxial layer from it, and another part of a solutionmelt becomes saturated. When feeding the next discrete portion $\mathrm{P}_{2}$ of a solution-melt over the previous one, the layer initially grows due to isothermal epitaxy initiated by mixing the first and second portions of melts, and after alignment of their structures there take place a growth caused by the compulsory cooling to $T_{2}=T_{1}-T_{2}$. This way of growing the epitaxial layers can be 
considered as a modification of delta-thermal liquidphase epitaxy in the multistage process. Thus, more stationary crystallization front is provided, and as though growth of each micro-layer is carried out with variable speed, more exactly with decreasing the speed. For initial crystallization temperature $T_{\mathrm{cr}}=750$ to $830^{\circ} \mathrm{C}$, optimum $\Delta T$ is within the range 3 to $10{ }^{\circ} \mathrm{C}$.

It is offered to achieve the accuracy to control the structure of grown epitaxial layers by feeding the solution-melt as discrete portions in accord with the law [4]

$$
V=m t^{0}\left[\left(t / t^{0}\right)^{m}-\left\{\left(t / t^{0}\right)-1\right\}^{n}\right],
$$

where $0 \leq n \leq 2, m$ - mass of one discrete part, $t$ - time of its introduction; and $m \leq 5 \cdot 10^{-2}$ mass of the basic solution-melt.

The advantage of the offered way is that all the amount of a solution-melt moves onto the substrate not at once but by portions.

This feeding way allows to gradually avoid the super-saturation and, in such a manner provides a controllable growth of the epitaxial layer. If feeding all the amount in one time, the growth rate would be very high in the very beginning, and then sharply decrease. In our case, each portion has the same super-saturation degree. As a result, the growth rate remains constant from the beginning up to the end of the process. As a result, the quality of layers and morphology of a surface from layer to layer become more and more perfect.

The choice of $\Delta T$ in the interval 3 to $10^{\circ} \mathrm{C}$ at the initial crystallization temperature $750 \ldots 855^{\circ} \mathrm{C}$ has allowed to obtain the epitaxial layers of $\mathrm{GaAs}$, InGaAs and AlGaAs with the thickness from 0.2 up to $2 \mu \mathrm{m}$ and containing from 4 to 8 micro-layers with a mirror upper surface.

When growing the epitaxial layers of solid solutions of aluminium-gallium arsenide on substrates of gallium arsenide, the following regularity was observed. The epitaxial layers grown up by compulsory cooling from solutions-melts $(\mathrm{Al}+\mathrm{Ga}+\mathrm{GaAs})$ of certain structure have at the boundary with a substrate the structure originally given in the melt, where the aluminium concentration decreases when coming to the surface of the layer.

If layers of solid solutions aluminium-gallium arsenide are grown by pressing out of the previous solution-melt with increasing amount of aluminium in the subsequent one, their structure turns out with an increased concentration of aluminium [5], that is the layers which are coming nearer to structure of each solution-melt grow on the boundaries of contacts with the solid phase.

Thus, at the growth of aluminium-gallium arsenide epitaxial layer the growing layer with bounding with a solid solution (substrate) watches (keeps up) to structure of a solution-melt created at front of crystallization. When the structure of grown aluminium-gallium arsenide layer is compared to the structure of a liquid solution-melt, the growth process stops. Accordingly, at the moment of a solution-melt contact with the substrate, the phase is formed on their boundary that is in equilibrium with the substrate, and, from which the growth of epitaxial layer begins at cooling or addition melt with structure distinct from the phase.

If to accept their stratification at the contact with the substrate as the basic difference of aluminium solutionmelts from indium or phosphorus ones, then at the initial moment the phosphorus solution-melt etches the surface of a substrate, and the indium one dissolves. According to certain critical structure, the phosphorus solution-melt will be stratified by layers with the large density of phosphorus at the solution-melt surface and with a smaller one at the substrate. On the contrary, the indium solution-melt will have the layers with the large indium density on the boundary with the substrate.

Let's note that in the case of indium solution-melts the temperature of wetting the substrate $(\mathrm{GaAs})$ is more effective than at the temperature of the solid solution growth. As a result, the gradient of the structure is created along the melt thickness during the growth process.

The structural ingomogeneity of solid solutions observable in practice as well as effects at the heteroboundary InGaAs-GaAs can be explained by the indium layer growth mechanism offered below.

At the initial moment of contacting between homogeneous solution-melt (In+Ga+GaAs) with a substrate (GaAs), there is etching the substrate (for example, dissolution, of one atomic substrate layer of a material with the removal arsenicum into the liquid bulk) with the creation of a superstructure. As a result, the equilibrium phase is formed at the boundary with a substrate as a thin sub-layer with the higher indium density and concentration, greater than in the solutionmelt bulk.

In the process of decrease of the system temperature, the difference in structures at the boundaries between solid and liquid phases is increased prior to the beginning of layer crystallization. The further growth of layers proceeds by moving the crystallization front into the depth of liquid with simultaneous formation of a solid indium-gallium arsenide solution of the same bulk structure. The reduction of indium and arsenicum in the volume of the melt is restored by removal of indium-arsenicum from the equilibrium sub-layer at the front of crystallization.

On the basis of thin $p-n$ junction grown up from a liquid phase on low-ohmic substrate with $p^{+}$-type conductivity the diode structures have been made. The area of a $n$-type is grown up in them from a source $(\mathrm{Ga}+\mathrm{GaAs})$ gallium-arsenide with the concentration of carriers $2 \cdot 10^{16} \mathrm{~cm}^{-3}$, and also has the thickness of 0.6 to $0.8 \mu \mathrm{m}$. The current-voltage characteristic of produced thin-junction diode structures have abnormal properties, as well as in structures with the Schottky barrier $[6,7]$. 


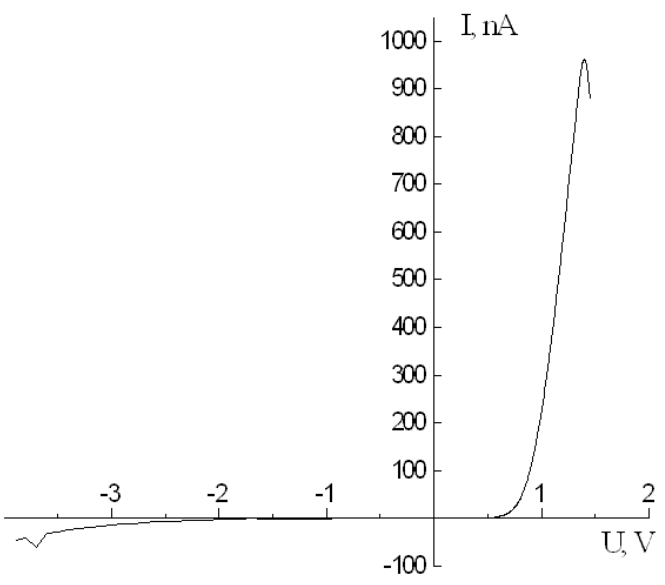

Fig. 1. Current-voltage characteristics of one of the structures.

The current-voltage characteristic data of one of the structures are given in Fig. 1. The current value decreases in the forward direction at the given voltage higher than $1.3 \mathrm{~V}$ and grows from $3.6 \mathrm{~V}$ in the opposite direction.

The abnormal photo-electric effect takes place also in the capacitor characteristics in the mode of a small variable signal influence.

The difference of the characteristic of the received structures is, that, they have selective photosensitivity, both from a working point, and from the frequency of a small variable signal. As shown in Fig. 2 the capacity of the $p-n$ junction in the darkness (curve 1) passes through the maximum at $5 \pm 0.5 \mathrm{~V}$. The greatest change in the capacity is observed at illumination just in this point (Fig. 2, curve 2). Up to this site the dependence of capacity on a cut-off voltage obeys the known law $C \sim \mathrm{V}^{2}$

Thus, the performed experiments show that the delta-thermal liquid epitaxy is suitable to obtain the thinlayer diode structures.

\section{References}

1. J.M. Woodall, Solution growth $\mathrm{Ga}_{1-\mathrm{x}} \mathrm{Al}_{\mathrm{x}} \mathrm{As}$ superlattice structures // J. Cryst. Growth 12 (1), p. 32-38 (1971).

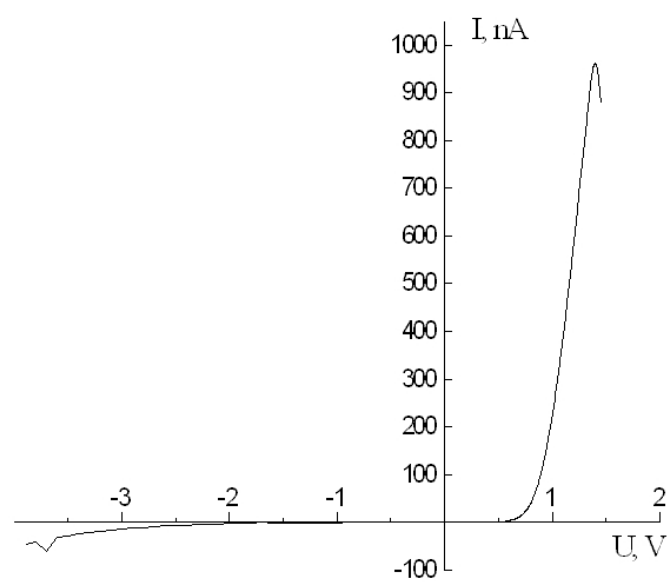

Fig. 2. Voltage-capacity characteristics (curve 1 - in darkness, curve 2 - at illumination).

2. P. Swapup, R.K. Jain, S.N. Verma, S. Charan, D.M. Tandle, Liquid epitaxial growth of layers AlGaAs of (100) GaAs //Ibid. 63 (1), p. 97-104 (1983).

3. Y.K. Su, C.C. Wei, S.C. Lu, C.C. Chang // Surf. Mater. Sci. 8 (1), p. 29-38 (1986).

4. A.V. Karimov, Sh. Mirtursunov, Method of epitaxial growing semiconductor compounds $\mathrm{A}^{3} \mathrm{~B}^{5}$. Patent SU N 1037795 (1983).

5. J.I. Alferov, V.M. Andreev, Liquid epitaxy heterojunction structures in system AlGaAs // Growth Semiconductor Crystals and Films 1, p. 209 (1977).

6. E.V. Vladimirova, K.A. Juravlev, V.A. Ivanchenko, V.I. Semenov, Volt-current characteristic of thinbase diode with Schottky barrier // Physics and Technics of Semiconductors 20 (10), p. 1905-1907 (1986).

7. N.V. Gorev, S.A. Kostilev, T.V. Makarova, E.F. Prohorov, A.T. Ukolov, Non-smoothness of voltcapacity dependence of thin-film semiconductor structures with Schottky barrier // Ibid. 23 (2), p. 357-359 (1989). 